\title{
Investigation of Atrial Vulnerability by Analysis of the Sinus Node EG From Atrial Fibrillation Models Using a Phase Synchronization Method
}

\author{
Ying Chen*, Zhong Wu*, Cuiwei Yang*, Jun Shao, Kelvin Kian Loong Wong, and Derek Abbott, Fellow, IEEE
}

\begin{abstract}
Atrial fibrillation (AF) can result in life-threatening arrhythmia, and a clinically convenient means for detecting vulnerability remains elusive. We investigated atrial vulnerability by analyzing the sinus electrogram (EG) from AF animal models using a phase synchronization method. Using acetylcholine (ACh)-induced acute canine AF models $(n=4)$, a total of 128 electrical leads were attached to the surface of the anterior and posterior atria, and the pulmonary veins to form an electrocardiological mapping system. ACh was injected at varying concentrations with ladder-type adjustments. Sinus EGs and induced AF EGs that pertain to specific ACh concentrations were recorded. We hypothesize that the atrial vulnerability may be correlated with the Shannon entropy (SE) of the phase difference matrix that is extracted from the sinus EG. Our research suggests that the combination of SE with the synchronization method enables the sinus node EG to be analyzed and used to estimate atrial vulnerability.
\end{abstract}

Index Terms-Atrial fibrillation (AF), electrophysiological mapping, phase synchronization, Shannon entropy, sinus rhythm.

\section{INTRODUCTION}

A TRIAL fibrillation (AF) is the most common type of arrhythmia and contributes to worldwide cardiovascular morbidity and mortality [1]. It can cause complications such as congestive cardiac failure and stroke [2]. Treatment of AF is a clinical challenge as the exact electrophysiological bases of $\mathrm{AF}$ initiation and maintenance are still not fully understood [3]. At present, methods used to understand and analyze AF range from gene expression and protein ion channels [4], [5] to electrocardiological mapping of epicardium and torso [6], [7]. Sanders et al. [7] identified sites of high-frequency activity and dealt with the diagnosis of $\mathrm{AF}$ and ablation as a surgical solution [8]. Oakes

Manuscript received August 6, 2011; revised December 2, 2011, March 7, 2012, May 23, 2012 and July 4, 2012; accepted July 5, 2012. Date of current version August 16, 2012. Asterisk indicates corresponding author.

${ }^{*}$ Y. Chen is with the Department of Electronic Science and Engineering, Nanjing University, Nanjing 210093, China (e-mail: chenying_nju@ hotmail.com).

${ }^{*} \mathrm{Z}$. Wu is with the Department of Thoracic and Cardiovascular Surgery, Nanjing Drum Tower Hospital, The Affiliated Hospital of Nanjing University Medical School, Nanjing 210008, China (e-mail: zhongwu@nju.edu.cn).

${ }^{*} \mathrm{C}$. Yang is with the Department of Electronic Engineering, Fudan University, Shanghai 200433, China (e-mail: yangcw@ fudan.edu.cn).

J. Shao is with the Department of Thoracic and Cardiovascular Surgery, Nanjing Drum Tower Hospital, The Affiliated Hospital of Nanjing University Medical School, Nanjing 210008, China (e-mail: shaojun1979nj@yahoo.com.cn).

K. K. L. Wong is with the RMIT University, Melbourne, Vic. 3001, Australia (e-mail: kwong@eleceng.adelaide.edu.au).

D. Abbott is with the School of Electrical and Electronic Engineering, Adelaide University, Adelaide, S.A. 5005, Australia (e-mail: dabbott@eleceng.adelaide.edu.au).

Digital Object Identifier 10.1109/TBME.2012.2208751 et al. [9] reported the utility of delayed-enhancement magnetic resonance imaging (DE-MRI) in detecting abnormal atrial tissue before radiofrequency ablation and in predicting procedural outcome.

The sinus electrogram (EG) has been used previously to evaluate ischemia [10] and to predict ischemic recoverability [11]. However, recently there have been many studies using the sinus EG to analyze the mechanisms of AF. Kneller et al. [12] and Nattel et al. [13] analyzed AF maintenance through the action potential (AP) and effective refractory period (ERP) values induced by acetylcholine (ACh), using a patch clamp and computer model. Shivkumar et al. [14] concluded that parasympathetic activation during $\mathrm{AF}$ ablation is associated with the presence of a preablation high-amplitude fractionated EG in sinus rhythm via a waveform identification method. In our study, we aim to analyze the atrial vulnerability through the sinus EG of a canine AF model.

It is well known that the myocardium usually operates in rhythm with the sinus node. We can adopt a simplified view that the EGs of the normal myocardium are linearly controlled by the EG of the sinus node mostly. When AF is in progress, the EG of the heart system becomes more disordered and random. We hypothesize that the atrium operates with the sinus node almost synchronously under a sinus rhythm and almost asynchronously before AF. In order to estimate synchronization in sinus EGs, we adopted entropic analysis and synchronization methods [15], [16] that are applied to a multielectrode ECG mapping system in order to obtain a full view of the disordered patterns resulting from the heterogeneous tissue.

First, acute canine AF models that are similar to those by Nishida et al. [1] are prepared. Ladder-type ACh concentrations are applied for controlling the degree of AF vulnerability. Additionally, anatomical modeling, which is based on the technique of medical image reconstruction during AF assessment [17], can be performed for electrocardiological map presentation and analysis.

\section{Methods}

\section{A. Experimental Procedures}

Animal studies were approved by the Animal Ethics and Experimentation Committee of the Nanjing University, Nanjing, China. The experiments were performed in accordance with the Guide for the Care and Use of Laboratory Animals that is published by the National Institutes of Health (publication no. 85-23, revised in 1996). 


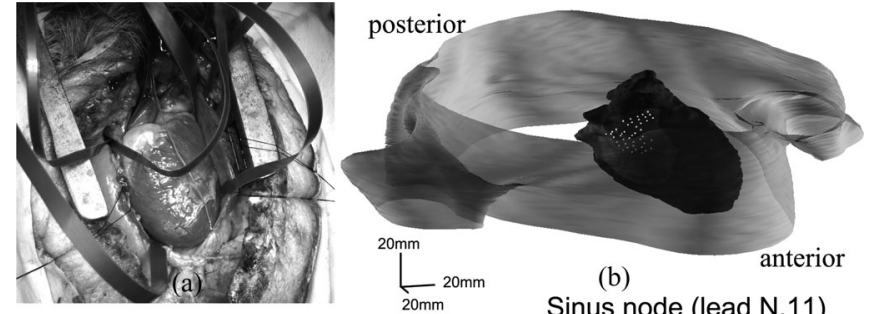

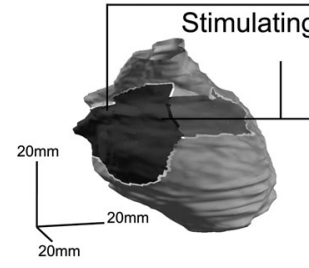

(c)
Sinus node (lead N.11) stimulating position
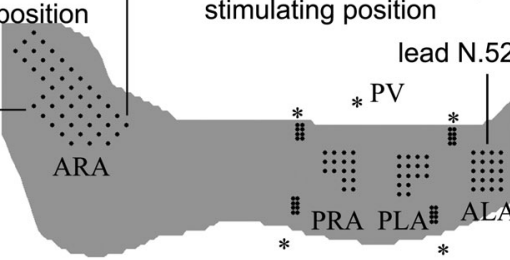

lead N.52

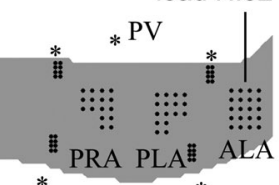

(d)

Fig. 1. Electrocardiological mapping containing a total of 128 electrode leads during AF. (a) In the electrocardiological mapping setup, eight epicardial patches with a total of 128 electrode leads were connected to the mapping system. (b) Locations and geometry of the atria are indicated by the 3-D reconstruction of the torso surface and epicardium model. (c) Atrial regions are highlighted by the red surface within the translucent heart representation. (d) Surface of the atrial model is spatially transformed into a 2-D plane with the labeling of the electrode lead locations, the sinus node, and two pairs of electrode stimulus locations. The waves and phase maps of lead numbers 11 and 52 are registered. The patches were rolled and placed around the PV. Their positions are illustrated in (d). (Note abbreviations: ARA, anterior right atrium; PRA, posterior right atrium; ALA, anterior left atrium; PLA, posterior left atrium; and PV (*), pulmonary veins).

Canine mongrels with weight $14.2 \pm 3.6 \mathrm{~kg}(n=4)$ were injected with ACh. After thoracotomy and suspension of the capsula cordis, the heart was exposed for placement of an electrocardiological mapping system [see Fig. 1(a) and (b)]. Electrodes were placed around the aortic root, which served as an electrical reference ground. We also registered right ventricular signals via a reference electrode. Two pairs of stimulating electrodes were placed on the right atrium and the interface between the superior vena cava and the right atrium [see Fig. 1(c) and (d)]. Pairs of stimulating electrodes, with leads separated by a distance of approximately $0.5 \mathrm{~cm}$, were connected to a program stimulator. Eight patches comprising 128 electrodes in total were sutured onto the surface of the anterior and posterior left atrium and right atrium, and pulmonary veins (PVs) [see Fig. 1(d)]. Unipolar electrodes, $1.2 \mathrm{~mm}$ in diameter and $\sim 3.5 \mathrm{~mm}$ to $5.0 \mathrm{~mm}$ apart, were carefully arranged in these flexible patches to match the size of the canine atrium in a surgical setting. Four flexible patches were rolled and placed around the PV sleeves. All 128 electrodes were connected to the FDEMS-2 128-channel epicardial mapping system [18] that was developed at the Instrumentation Laboratory, Fudan University, Shanghai, China. Reconstructed CT images show the mapped atrial region for one canine heart in Fig. 1(b) and (c).

A vein catheter was connected with a micropump that continuously injected $2 \mathrm{~g} / \mathrm{L}$ of $\mathrm{ACh}$ solution at a rate of $10 \mu \mathrm{g} \cdot \mathrm{kg}^{-1} \cdot \mathrm{min}^{-1}$. Before stimulus, we recorded the EG mapping signals for $3 \mathrm{~min}$. To induce $\mathrm{AF}$, a burst-pacing stimulus at frequency of $20 \mathrm{~Hz}$, pulse width of $2 \mathrm{~ms}$, and duration of $5 \mathrm{~s}$ was transmitted to the stimulating electrodes. The voltage of the burst waves was twice that of the pacing threshold. The burst waves were repeated five times and the induced AF occurrence times and durations were recorded. According to the $\mathrm{AF}$ times and durations, the $\mathrm{ACh}$ concentration was increased stepwise and the stimulation and recording were repeated until a maximum $\mathrm{AF}$ condition was achieved. A maximum $\mathrm{AF}$ condition was defined to be when $>3$ min duration of AF could be obtained each time by five programmed burst stimulations. The potential AF duration of sinus EG was then labeled by its corresponding ACh concentration. As AF is induced by electrical stimulation, the complex AF initialization is not included in the data analysis. So, only the induced AF duration that is due to the affect of ACh serves as an indicator of atrial vulnerability.

\section{B. Signal Acquisition}

At the beginning of every experiment, we ensured that the ACh was injected into the body for more than $3 \mathrm{~min}$ in order for the heart to become accustomed to its chemical effect. Then, we measured the sinus EG for another $3 \mathrm{~min}$. We implemented a ladder-type variation of $\mathrm{ACh}$ concentrations to induce $\mathrm{AF}$ after electrical activation of the heart. Every heart model experiences ACh concentration increments from zero to the peak, and then reverses back to zero again. Although the experiments on canine AF models can take up to $9 \mathrm{~h}$, AF could still be induced until the end of the experiment. Up to 15 datasets of EGs based on varying $\mathrm{ACh}$ concentrations were acquired for each model. With the data of 128 electrode leads acquired simultaneously, each experiment generates up to $5 \mathrm{~GB}$ of information.

\section{Signal Preprocessing}

The EG mapping system consists of 128 isolated amplifier channels with adjustable gain (25-2000) and a fixed bandwidth $(3-500 \mathrm{~Hz})$. The $16-b i t$ digital values were sampled at $2 \mathrm{kHz}$. The data included 127 atrial signals and a ventricular signal as a reference. Due to the higher voltage for the ventricular waves and the intense periodicity of sinus EGs, the influence of heart rate and disturbance of ventricular waves were removed by applying a preprocessing stage across all signals. Through the ventricular reference electrode, $R$ peak locations were detected using a wavelet transform, and the mean heart rate and its standard deviation (STD) were obtained. Rectangular windows of 350 points $(175 \mathrm{~ms})$ in duration were applied between 195 and $20 \mathrm{~ms}$ in advance of the R peaks. The preprocessed signals of the same signal length included an equal cardiac cycle without the disturbance of ventricular waves and heart rate [see Fig. 2(a) and (b)]. Finally, the preprocessed signals were filtered by a lowpass zero-phase frequency impulse response (FIR) filter using a $175-\mathrm{ms}$ Hamming window. The cutoff frequency $\left(F_{h}\right)$ was changed from 100 to $20 \mathrm{~Hz}$ in order to analyze the results and to remove high-frequency random fluctuations in the signals. The results are based on a data length of 40000 points that include 147 cardiac cycles.

A proportion of the signals had low signal-to-noise ratio (SNR), which were typically due to poor electrical contact with the atrial tissue. In addition, the size of the canine hearts made 


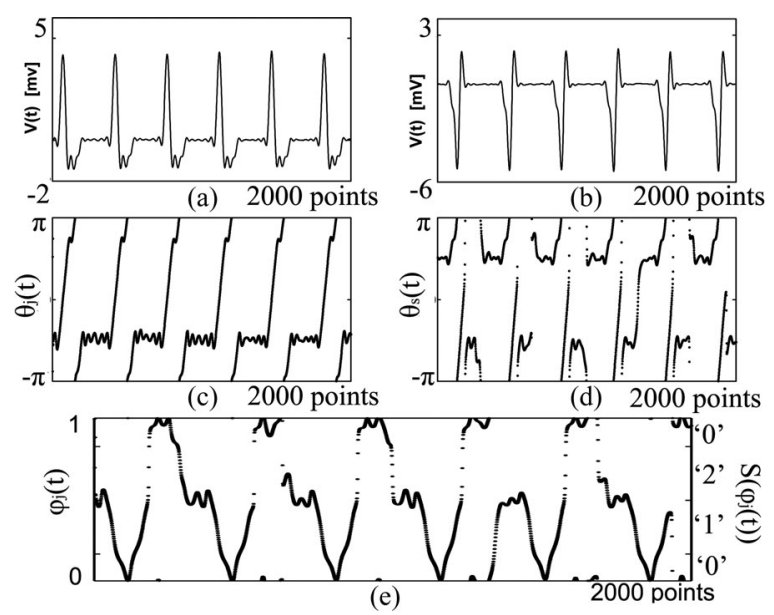

Fig. 2. Analysis of sinus EG. The EG signals utilize 2000 points of data and are preprocessed in order to remove the disturbance of ventricular waves and heart rate. Voltage signals $V(t)$ of leads N.52 (a) and N.11 (b) were filtered by a zero-phase FIR filter $(3-60 \mathrm{~Hz})$. We applied the Hilbert transform to obtain the phase $\theta_{j}(t)$ of N.52 (c) and $\theta_{s}(t)$ of N.11 (d). After unwrapping the $\theta$ signals, we performed further signal processing to obtain the phase difference $\varphi_{j}(t)$ (e). Finally, the symbolic method was applied and the symbolic $\varphi_{j}(t)$ was obtained as $S\left(\varphi_{j}(t)\right)$.

it difficult to set up the electrode patches. Only signals with a similar heart rate as that of the ventricular reference signals were selected.

\section{Dominant Frequency $(D F)$ Map of $A F E G$}

The DF is defined as the frequency of the dominant peak in the power spectrum [6], [7], [19], [20], and computed for each electrode signal using a fast Fourier transform with a rectangular window size of $10 \mathrm{~s}$ in order to provide a frequency resolution of $0.1 \mathrm{~Hz}$. The spectrogram can be used to represent each signal in the time-frequency domain [6]. To control the ambiguity in DF detection that is related to low SNR, the regularity index (RI) [7] was determined. Only points with RI $>0.2$ were included in the subsequent analysis.

\section{E. Time Delay Difference Map of Sinus EG}

In order to examine the effect of $\mathrm{ACh}$, atrial epicardial activation times were calculated using the steepest negative intrinsic deflection of each epicardial EG during the activation complex (i.e., minimum $d V / d t$ ). The mean time delay of activation waves passing from the sinus node to other electrodes was then determined. The conduction velocity is inversely proportional to the time delay. A time delay difference map was obtained by subtracting the time delay map of optimal and zero ACh concentrations. Positive values in the time delay difference imply that their conduction velocities are slowed down.

\section{F. Signal Analysis}

Our basic approach is derived from the phase synchronization method [15], [16], which we then apply to evaluate synchronized atrial excitation. In addition, we use Shannon entropy (SE) [21] to assess the randomness of atrial synchronization.
We assume that the EG at the sinus node is periodically controlled by this node. As such, periodic analysis of the atrial activity was implemented. First, the voltage matrix $V$, which represents the voltage time series of all the electrodes, is constructed by the number of atrial electrodes and data length of time $N$ (40 000 points). The phase matrix $\theta$ is obtained by applying a Hilbert transform [22], [23] to each row of the $V$ matrix (see Fig. 2). The phase difference matrix $[\varphi(t)](1 \leq t \leq N)$ is constructed by $\theta_{j}(t)$ [see Fig. 2(c)] or each row of $\theta$ minus $\theta_{s}(t)$ $[s=11$ in Fig. 2(d)] for the electrode near the sinus node [see Fig. 2(e) $]$, as $\varphi_{j}(t)=\left[\left(\theta_{j}(t)-\theta_{s}(t)\right)(2 \pi)^{-1}\right] \bmod 1(1 \leq j \leq 128$, $1 \leq t \leq N)$. The SE of the phase difference matrix [21], [23], which is given by $[\varphi(t)]$, can then be determined.

We let the phase difference matrix $[\varphi(t)]$ represent a time vector series length of $N$ points. Each vector $\varphi(t)$ is composed of a normalized phase difference that pertains to every electrode at the same time $t$ such that $\varphi(t)=\left[\varphi_{1}(t), \ldots, \varphi_{\mathrm{j}}(t), \ldots, \varphi_{128}(t)\right],(0 \leq j \leq$ $128,1 \leq t \leq N)$. Then, we performed the Piecewise Aggregate Approximation (PAA) [24] symbolic method and transform $\varphi_{j}(t)$ to $S\left(\varphi_{j}(t)\right)$, in order to reduce the computation time where

$$
\begin{aligned}
& S\left(\varphi_{j}(t)\right)= \begin{cases}2, & 1 / 2 \leq \varphi_{j}(t)<5 / 6 \\
1, & 1 / 6 \leq \varphi_{j}(t)<1 / 2 \\
0, & 0 \leq \varphi_{j}(t)<1 / 6,5 / 6 \leq \varphi_{j}(t) \leq 1\end{cases} \\
& (1 \leq j \leq 128,1 \leq t \leq N) .
\end{aligned}
$$

Due to the $2 \pi$ period of the phase space, phase values near zero and $2 \pi$ were transformed to the symbol " 0 ." The remaining phase values were equally separated and transformed to symbols "1" and " 2 ." Through the symbolic method of (1), the matrix $[\varphi(t)]$ is transformed to $S\left(\varphi_{j}(t)\right)=\left[S\left(\varphi_{1}(t)\right), \ldots, S\left(\varphi_{128}(t)\right)\right]$ [see Fig. 2(e)]. The SE of the symbolic phase difference matrix $[\varphi(t)]$ is calculated as follows [21]:

$$
p(s(\varphi(t))=\operatorname{Prob}\{S(\varphi(k))=S(\varphi(t)), \quad 1<k \leq N\}
$$

$$
\mathrm{SE}=-\sum_{j=1}^{N} p(S(\varphi(t))) \log p(S(\varphi(t))) \quad(1 \leq t \leq N)
$$

Here, the SE reflects the disorder in the sequence represented by the vector $\varphi(t)$. We hypothesize that the phase difference matrix $\varphi$ contains information on the synchronous mode of the atrial electrical activity. Then, the SE of the matrix $[\varphi(t)]$ was used to evaluate the uncertainty in synchronization or the randomness in the atrial synchronization [25]. The dimensions of $\varphi$ are the number of atrial electrodes and the data length $N$ (40 000 points).

\section{RESULTS}

\section{A. Statistical Analysis}

The maximum ACh concentration was defined to be that for which $\mathrm{AF}$ with duration $>3$ min could be induced at least 
TABLE I

MEAN VALUES AND STD OF ACQUIRED Sinus HeART RATE

\begin{tabular}{llll}
\hline \hline & Normal state & Peak state & End state \\
\hline ACh $\left(\mu \mathrm{g} \cdot \mathrm{kg}^{-1} \cdot \mathrm{min}^{-1}\right)$ & 0 & $81 \pm 23$ & 0 \\
Mean heart rate $(/ \mathrm{min})$ & $85.2 \pm 16.4$ & $75.2 \pm 23.5$ & $74.3 \pm 27.4$ \\
STD heart rate $(/ \mathrm{min})$ & $1.6 \pm 01.0$ & $4.0 \pm 1.1$ & $4.3 \pm 0.5$ \\
\hline
\end{tabular}

The models are analyzed based on 3 levels of acetylcholine (ACh) concentrations using the following parameters: 1) ACh concentrations, where we note that the peak ACh state corresponds to the maximum atrial fibrillation duration; 2) Heart rate mean; and 3) Heart rate standard deviation (STD).

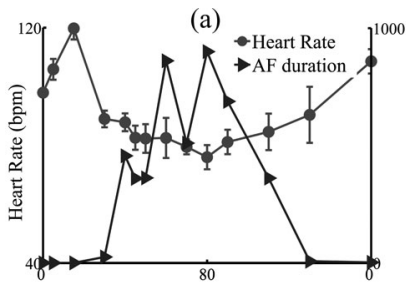

(c)
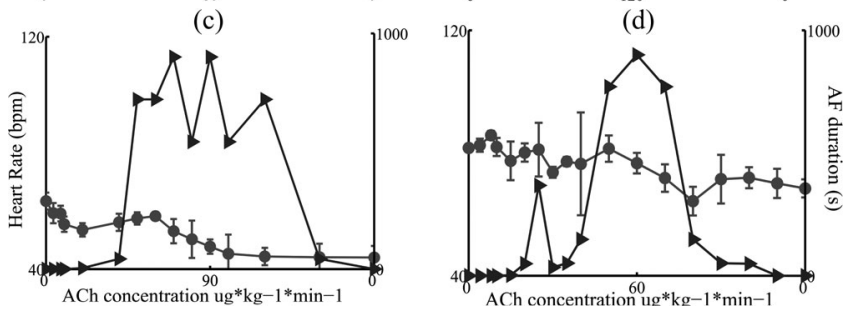

Fig. 3. Analysis of induced AF duration (s) and heart rates (beats/min). Canine model (a) No. 1 (Female and $11 \mathrm{~kg}$ ), (b) No. 2 (Male and $15 \mathrm{~kg}$ ), (c) No. 3 (Male and $12 \mathrm{~kg}$ ), and (d) No. 4 (Male and $13 \mathrm{~kg}$ ) were used. No ischemia was observed for experiment No. 1 and No. 2. But ischemia occurs for No. 3 and No. 4, which were revived by oxygen supply. After preprocessing, $30 \pm 9$ erroneous electrodes were removed. The ACh concentration was incremented to max values and then decreased. The induced AF duration together with the mean heart rate and STD versus ACh map is then produced. Peak ACh concentration is $81 \pm 23 \mu \mathrm{g} \cdot \mathrm{kg}^{-1} \cdot \mathrm{min}^{-1}$ and the first long AF concentration is $41 \pm 14 \mu \mathrm{g} \cdot \mathrm{kg}^{-1} \cdot \mathrm{min}^{-1}$.

five times. The maximum concentrations were $80,120,70$, and $60 \mu \mathrm{g} \cdot \mathrm{kg}^{-1} \cdot \mathrm{min}^{-1}$ for the four animal case studies used. The first occurrence of induced $\mathrm{AF}$ greater than 3 min corresponded to an ACh concentration of $41 \pm 14 \mu \mathrm{g} \cdot \mathrm{kg}^{-1} \cdot \mathrm{min}^{-1}$. Table I presents the different states of ACh concentration and the statistics of their corresponding sinus EG, which includes the heart rate's mean and STD values.

\section{B. Duration of Induced AF and Heart Rate}

The induced AF duration versus ACh graphs (see Fig. 3) are based on ACh that was injected at varying concentrations with ladder-type adjustments. The results show that the AF duration is influenced by the ACh concentration. The AF duration curves possess greater fluctuations during the $\mathrm{ACh}$ increment process than the ACh decrement process. Upon reaching the maximum $\mathrm{ACh}$ concentration together with an optimal AF duration, we increased the ACh concentration and observed the resulting irregular AF maintenance (see Fig. 3(c)]. The mean and STD values that pertain to the heart rate are obtained via analysis of the sinus EG.

\section{Electrochemical Mechanisms of AF Induced by ACh}

Both the direct stimulation and application of ACh are reported to induce AF experimentally [6], [13]. The method involves infusing the ACh ladder-type concentrations into the blood flowing toward the heart and then applying electrical stimulation at the tip of the right atrium to cause AF. The fibrillation was then maintained as long as sufficient concentration of ACh was infused since the single-cell AP duration, myocardial conduction velocity, and ERP are shortened due to the affect of $\mathrm{ACh}$ [12]. Fibrillation was observed in different experiments for periods up to 30 and $60 \mathrm{~min}$. When the fibrillation stops, it can always be restarted by the same procedure. Thus, the fibrillation is not a transitory phenomenon. The fibrillation must therefore be a manifestation of ACh action on the heart.

The DF map and time delay difference map of canine model No.1 during the optimal ACh concentration $\left(80 \mu \mathrm{g} \cdot \mathrm{kg}^{-1} \cdot \mathrm{min}^{-1}\right)$ are shown in Fig. 4. Time delay difference maps are present in between the optimal and the zero ACh concentrations.

\section{SE Maps}

We now analyze the sinus EG data and compare it against ACh concentrations and different AF durations. Note that the cutoff frequency and the number of electrodes $\mu$ should be determined in advance.

1) Based on the adjustment of the cutoff frequency that pertains to the zero-phase low-pass filter, we analyze the frequencies of the EG signals and their influence on the results in Fig. 5(a). The SE values increase with the bandwidth of the sinus EG, which accords with expected information theory [26]. The parameter $\mu$ is fixed to 90 . When the cutoff frequency reaches $20 \mathrm{~Hz}$, the result is improved without changing the waveforms for the best contrast. This means the ACh effect mainly exists at low frequency, which is consistent with the shortened AP of a single cell by the ACh effect [12].

2) As $\mu$ was adjusted, the vector size of the phase difference matrix $\varphi(t)$ changed accordingly. The cutoff frequency is fixed at $20 \mathrm{~Hz}$. After low SNR channels were removed from the dataset, we calculated the maximum values $C_{\max }$ of the cross-correlation sequence that pertain to sinus EG between any electrode and the sinus node. We selected the first $\mu$ number of electrodes whose mean values of $C_{\max }$ were ranked in increasing magnitude. The $\mathrm{SE}$ and AF duration versus the ACh curves are shown in Fig. 5(b) with different $\mu$. The SE values increase gradually with $\mu$ when the cutoff frequency is fixed. This result indicates that (A) the atrial sites, where the EG is dissimilar to the sinus node EG, contribute more to the atrial synchronization, and (B) $\mu$ should be fixed for contrasting the atrial synchronization.

Since $\mu$ obviously affects the SE values, we calculated the mean SE values with respect to $\mu$ changing from 10 to 90 instead of a fixed number. The AF duration and SE values are fitted by a Gaussian function. Fig. 6(a)-(d) shows all the canine AF models. The fitted curves show that the ACh increment process increases the $\mathrm{AF}$ duration and $\mathrm{SE}$; the decremented $\mathrm{ACh}$ process 
(a)

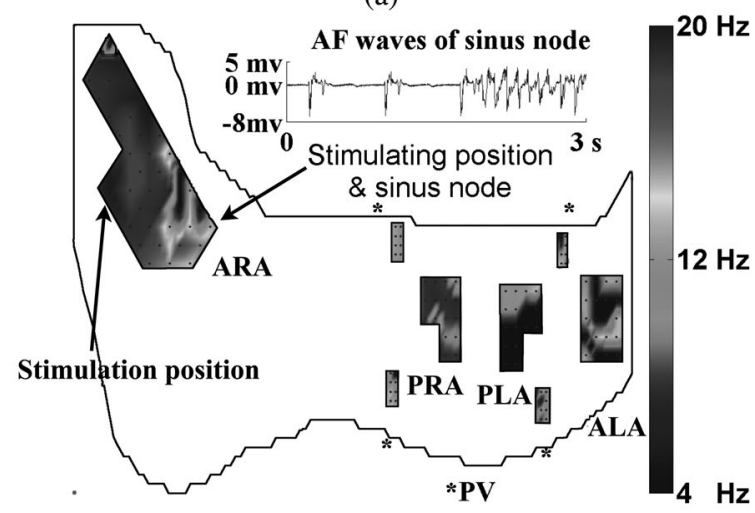

(b)

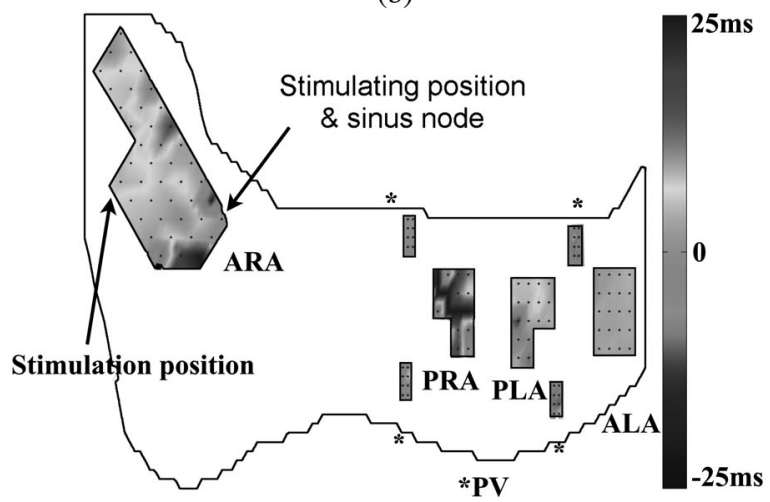

Fig. 4. DF map and time delay difference map of canine model No. 1, during the optimal ACh concentration $\left(80 \mu \mathrm{g} \cdot \mathrm{kg}^{-1} \cdot \mathrm{min}^{-1}\right)$. (a) Mean DFs are computed from the EG signals during AF by rectangular windows of $10 \mathrm{~s}$. The spontaneous AF wave onset of the sinus node is also shown in (a) as an example. (b) Time delay difference map between optimal ACh concentration and zero ACh concentration is calculated from difference values of mean time delay of activation waves, which pass from the sinus node to the electrodes. The data between patches are scaled to an appropriate mid-level range, as these are not relevant. We observe that wave propagation speed slows down mainly on ARA and PRA (larger time delay difference values) and the DF sites mainly exist in the right atrium (higher frequency sites); moreover, stimulating positions are also on right atrium. (Note abbreviations: ARA, anterior right atrium; PRA, posterior right atrium; ALA, anterior left atrium; PLA, posterior left atrium; and $\mathrm{PV}(*)$, pulmonary veins.)

illustrates that the SE decreases with the AF duration. Note that for some ACh concentrations, the SE values are unstable in Fig. 6(c), which is possibly caused by the fluctuations of $\mathrm{AF}$ duration if the $\mathrm{ACh}$ concentration continues to increase after the AF duration reaching the maximum. This may also indicate that $\mathrm{AF}$ is a complex process that is driven by multiple features.

\section{DISCUSSION}

\section{A. ACh Effect on the Acute Animal Model}

Kneller et al. [12] reported that vagal actions were observed based on patch-clamp studies using an island of ACh. In the control experiments by Kneller et al. [12] and Nattel et al. [13], a single extra stimulus resulted in a highly meandering unstable spiral wave, with DF $6.5 \mathrm{~Hz}$, whereas ACh accelerated the DF

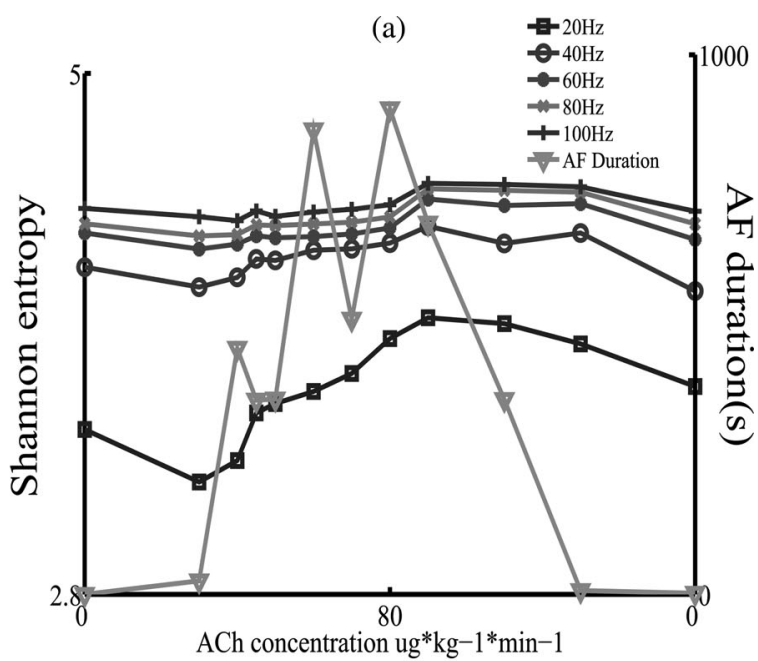

(b)

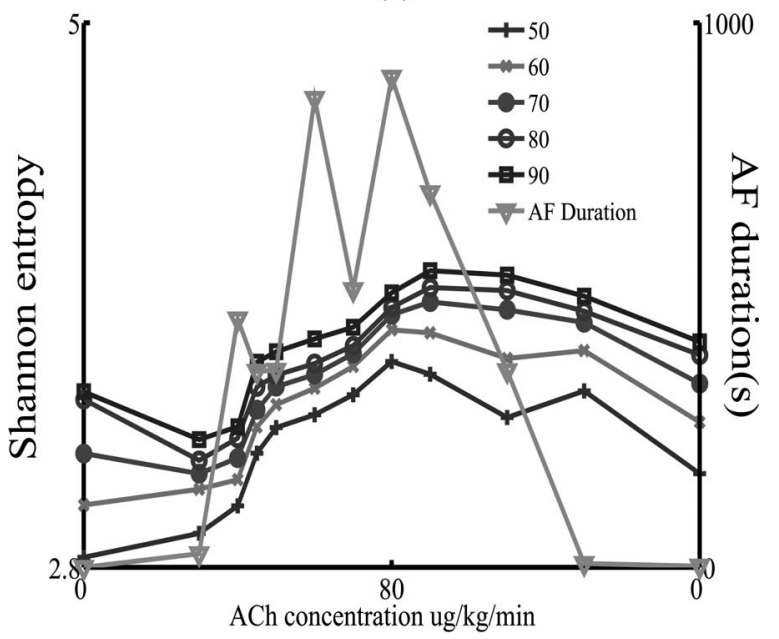

Fig. 5. Cutoff frequency effect and affect of electrode number on Shannon entropy. Canine model No. 1 was used. (a) SE versus ACh map with the cutoff frequency of zero-phase low-pass filters changing from 20 to $100 \mathrm{~Hz}$ is shown and the number of electrodes $\mu$ is fixed to 90. (b) SE versus ACh map with $\mu$ changing from 50 to 90 are shown and the cutoff frequency is fixed to $20 \mathrm{~Hz}$. The results show that both the cutoff frequency and $\mu$ will affect the SE values, but neither of them will affect the correlation between SE and ACh. The SE values increase and decrease with ACh concentration with a lower cutoff frequency and $\mu$. The $\mathrm{AF}$ durations are also shown for comparison.

to $17.0 \mathrm{~Hz}$. Our results also support their conclusion that vagal AF may arise from disorganization of the heterogeneous tissue response.

The DF and time delay dispersion are found to be heterogeneous. The highest DF on anterior right atrium (ARA) and posterior right atrium (PRA) is about $18 \mathrm{~Hz}$ (see Fig. 4) due to the high ACh concentration injected in the vein [7], [12]. There are sites with low DF (near zero), because the RI [7] is below the threshold in these sites. It is found that ACh influences ion channels such as the $\mathrm{G}$ protein-gated potassium channels, and reduces the excitation ability of the cardiac cells [27]. The delay difference map between the optimal and zero ACh concentrations was calculated [see Fig. 4(b)]. It shows a change in the mean time delay of activation waves, which is passed from the sinus node to the electrodes. The larger time delay difference 


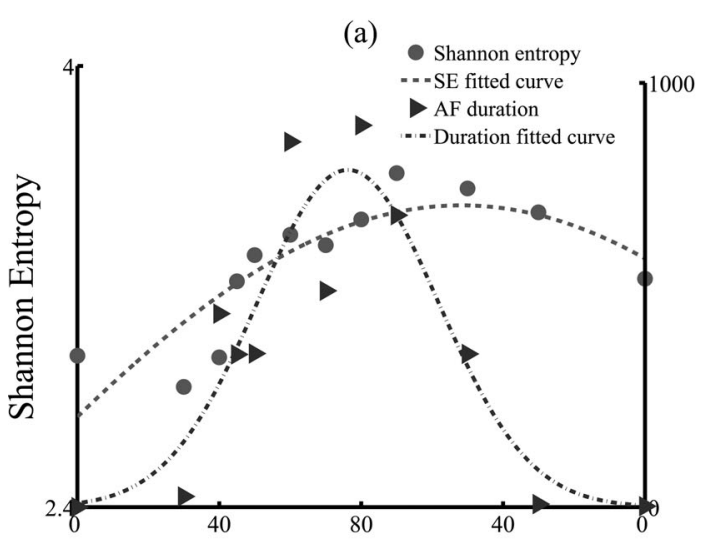

(c)

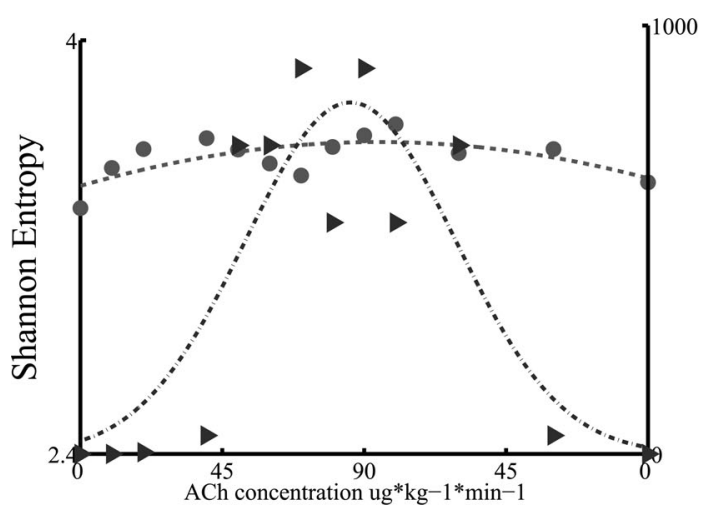

(b)

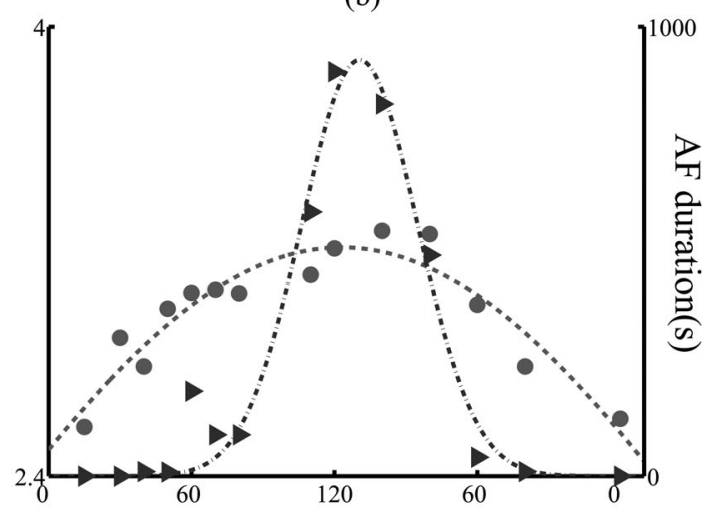

(d)

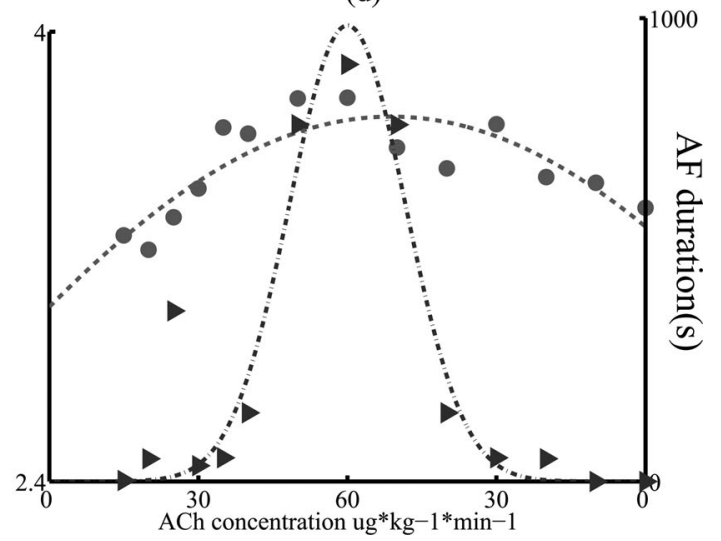

Fig. 6. Analysis of induced AF duration and SE mean values. (a) Canine model No. 1 (Female and $11 \mathrm{~kg}$ ), (b) No. 2 (Male and 15 kg), (c) No. 3 (Male and $12 \mathrm{~kg}$ ), and (d) No. 4 (Male and $13 \mathrm{~kg}$ ) were used. The cutoff frequency is fixed to $20 \mathrm{~Hz}$. The SE values are averaged according to the number of electrodes $\mu$ changing from 10 to 90 . The AF duration and SE values are fitted by a Gaussian function. STD errors of the fit were (a) 0.15, (b) 0.08, (c) 0.07, and (d) 0.1.

values indicate that conduction velocity slows down mainly on ARA and PRA.

The results in Figs. 3 and 4 demonstrate that it is possible to use a ladder-type concentration variation of ACh for controlling the degree of AF vulnerability. If there is a correlation between the AF duration and the synchronization method, their values versus ACh concentrations would be expected to show some correlation.

\section{B. SE With Synchronization Method}

The synchronization method was designed to give a view of the heterogeneous tissue response (synchronization of EG) based on different atrial vulnerability. The entropic analysis is capable of dimensionality reduction in the large EG phase difference database. Theoretically, with the SE, we could fully map the atrial synchronization if the system can map all of the atrium.

We hypothesize that the whole atrium operates with the sinus node almost in synchrony with the sinus rhythm and almost asynchronously before AF. Since the randomness of atrial synchronization (given by the SE) is inversely proportional to the synchronization, the synchronization behavior of the whole atrium working with the sinus node decreases when AF is developing and increases when AF is disappearing. The tendency for randomness in atrial synchronization, during sinus EG, may represent the atrial vulnerability. Our results examined this hypothesis and strongly suggested that there is correlation between atrial synchronization behavior and vulnerability.

By inspection of Fig. 6, it appears that the subjects can be ranked by their responsiveness to the ACh perturbation, with Fig. 6(d) reaching maximal levels of AF duration at only $60 \mu \mathrm{g} \cdot \mathrm{kg}^{-1} \cdot \mathrm{min}^{-1}$, and the others reach it at higher concentrations such as (C) 70 , (A) 80 , and (B) $120 \mu \mathrm{g} \cdot \mathrm{kg}^{-1} \cdot \mathrm{min}^{-1}$. This suggests that the atrial vulnerability of different subjects decreases in the same order. The SE values of the curves at $60 \mu \mathrm{g} \cdot \mathrm{kg}^{-1} \cdot \mathrm{min}^{-1}$ are approximately (D) 3.7 , (C) 3.6 (A) 3.33, and (B) 3.0. Note that when the subject obtains higher atrial vulnerability, higher SE values at a certain ACh concentration are observed. This motivates independent follow-up studies with a greater sample size and elucidates our initial hypothesis.

Peaks in the SE curves, which are shown by Fig. 6, are not consistent with those of the AF duration - the reason for this remains an interesting open question, which also motivates followup studies with greater sample size. Sometimes, when the ACh concentration goes to zero again, the SE values decrease but do not return to their initial values.

During the sinus EG, the atria synchronize with the sinus node. The synchronization behavior in the sinus EG gives an insight on how the sinus node generates impulses and passes 
along the heterogeneous substrate, considering that AF only occurs for certain heterogeneity conditions [12] and cholinergic effects are heterogeneously distributed in vivo [28]. We suggest that the SE with the synchronization method reveals the disorder in the activation phase vectors or rather the disorder of the heterogeneous atrial tissue response [28]. Hence, our method may possibly be applicable to different AF models such as chronic AF models. An AF model can be distinguished by the weak and random synchronization between sinus node and atria during sinus EG recordings. A normal heart tends to show a more regular impulse propagation that pertains to the atria.

\section{Effect of Parameters on SE of Sinus EG}

We implemented the SE and AF duration curves based on Gaussian curve fitting (see Fig. 6) in order to demonstrate their correlation and reduce signal fluctuations. In most of our case studies, the SE variation is observed to possess moderate fluctuations. The bandwidth of sinus EG, the electrode number, and selection method of electrode will also affect SE values. Due to the difference of individual hearts, the fluctuations of SE cannot be eliminated and the contrast between individuals may have no statistical significance.

\section{Comparison With Experimental Studies of Vagal AF}

Acute AF models using ACh were developed about 60 years ago. For instance, Burn et al. [29] reported the effect of ACh in the heart lung preparation including AF, and recently, Sanders et al. [7] induced the AF in an isolated sheep heart. The AF effects without ladder-type ACh concentration variations were performed in the same way in these articles. Kneller et al. [12] analyzed the AF maintenance induced by increasing ACh concentrations through different ways including, for example, the AP using patch clamp and computer model. The APD versus ACh curve decreases exponentially with ACh increment. In this study, we used both an increment and a decrement procedure.

Many methods have been developed or applied using the ACh model of AF, such as DF, AP, and ERP [6], [7], [19]. Some methods such as the DF method are only suitable for AF signals, whereas others such as ERP can only be performed with catheter measurements and are difficult to apply to the whole atrium. SE together with the synchronization method is developed and adopted in this paper for the first time. The synchronization method was used to examine the atrial synchronization behavior. The entropy analysis reduces the redundant information caused by multichannel signal processing. The entropy analysis method and the synchronization method applied to the multielectrode ECG mapping system can give a clear view of disorder resulting from a heterogeneous tissue response.

\section{E. Novel Findings}

The acute canine AF models are developed based on laddertype concentration variations of ACh for controlling the degree of AF vulnerability. This model can modulate the induced AF duration easily and repeatedly. The SE with a synchronization method is applied for the first time to epicardial signals and rep- resents a new way for analyzing AF via sinus EGs. The results demonstrate that atrial vulnerability can be monitored via the SE based on sinus EGs. It is possible to qualitatively estimate the atrial vulnerability via the atrial synchronization ability of the sinus EGs. In future, this method has the potential for postablation evaluation by a noninvasive ECG system with sufficient spatial resolution. Our method is designed to evaluate the synchronization ability of the atrium, and estimates the disorder in the heterogeneous atrial tissue response. It may be possible to investigate these common properties of AF using the synchronization method for the other models that pertain to different $\mathrm{AF}$ factors such as fibrosis, age, mutation, etc. More importantly, our study suggests that there is correlation between the atrial synchronization behavior and the atrial vulnerability.

\section{F. Limitations of the Study}

In addition to the ACh concentration, factors that affect the relationship of induced AF duration and SE values are the following: 1) electrical stimulation, which at the beginning of our experiment, will cause a significant change of SE value before and after the electrical stimulation even without ACh ejection; 2 ) ischemia, which is the main problem encountered in most animal experiments, will distort the results irreversibly and cause obvious reduction of the SE values; 3) drugs, such as anesthetics affecting heart rate, should be carefully used because of the uncertain influence on the affect of ACh; and 4) positive inotropic effects, which usually occur after the ACh stimulus is reduced rapidly, may cause some hysteresis in the SE curves (see Fig. 5). Without the interference of these factors, ACh concentrations are expected to modulate the induced AF duration and $\mathrm{SE}$ values.

Several limitations of our experiment exist. First, the study presented here was carried out in animal models representing acute AF. Second, our model is essentially a model of cholinergic AF. As such, the relevance of these data to human AF remains to be determined. Finally, the number of bipolar electrodes used in this study may not generate a sufficiently high volume of data for precise analysis.

\section{CONCLUSION}

We exploit the SE of the phase difference matrix from sinus EG via the influence of ladder-type ACh increments or decrements, and conclude that any random trend-indicated by the SE-pertains to atrial synchronization in the sinus EG and can qualitatively estimate the atrial vulnerability. Our scientific contribution is the potential elucidation of the basis of AF and predicting the atrial vulnerability using EGs.

\section{ACKNOWLEDGMENT}

The authors would like to thank X. Huang, Q. Zhan, and S. Yi from the School of Electronic Science and Engineering, Nanjing University, Nanjing, China, for their assistance in the preparation of this paper. 


\section{REFERENCES}

[1] K. Nishida, G. Michael, D. Dobrev, and S. Nattel, "Animal models for atrial fibrillation: Clinical insights and scientific opportunities," Europace, vol. 12, pp. 160-172, Feb. 2010.

[2] V. Fuster, "ACC/AHA/ESC 2006 guidelines for the management of patients with atrial fibrillation-executive summary: A report of the American College of Cardiology/American Heart Association Task Force on Practice Guidelines and the European Society of Cardiology Committee for Practice Guidelines (Writing Committee to Revise the 2001 Guidelines for the Management of Patients With Atrial Fibrillation)," Circulation, vol. 114, pp. E257-E354, Aug. 7, 2007.

[3] S. Nattel, "New ideas about atrial fibrillation 50 years on," Nature, vol. 415, pp. 219-226, Jan. 10, 2002.

[4] S. Nattel, S. Cardin, E. Libby, P. Pelletier, S. Le Bouter, A. ShiroshitaTakeshita, N. Le Meur, J. Leger, S. Demolombe, A. Ponton, and L. Glass, "Contrasting gene expression profiles in two canine models of atrial fibrillation," Circulation Res., vol. 100, pp. 425-433, Feb. 16, 2007.

[5] S. Makary, A. Maguy, R. Wakili, N. Voigt, K. Nishida, D. Dobrev, and S. Nattel, "Mechanisms underlying activation of constitutive acetylcholine-regulated potassium channels by atrial tachycardia remodeling," Circulation, vol. 120, pp. S664-S664, Nov. 3, 2009.

[6] R. Mandapati, A. Skanes, J. Chen, O. Berenfeld, and J. Jalife, "Stable microreentrant sources as a mechanism of atrial fibrillation in the isolated sheep heart," Circulation, vol. 101, pp. 194-199, Jan. 18, 2000.

[7] P. Sanders, O. Berenfeld, M. Z. Hocini, P. Jais, R. Vaidyanathan, L. F. Hsu, S. Garrigue, Y. Takahashi, M. Rotter, F. Sacher, C. Scavee, R. PloutzSnyder, J. Jalife, and M. Haissaguerre, "Spectral analysis identifies sites of high-frequency activity maintaining atrial fibrillation in humans," Circulation, vol. 112, pp. 789-797, Aug. 9, 2005.

[8] P. Sanders, C. Scavee, P. Jais, and M. Haissaguerre, "Left atrial pacemaker dominance after catheter ablation of atrial fibrillation," J. Cardiovasc. Electrophysiol., vol. 15, pp. 1324-1327, Nov. 2004.

[9] R. S. Oakes, T. J. Badger, E. G. Kholmovski, N. Akoum, N. S. Burgon, E. N. Fish, J. J. Blauer, S. N. Rao, E. V. DiBella, N. M. Segerson, M. Daccarett, J. Windfelder, C. J. McGann, D. Parker, R. S. MacLeod, and N. F. Marrouche, "Detection and quantification of left atrial structural remodeling with delayed-enhancement magnetic resonance imaging in patients with atrial fibrillation," Circulation, vol. 119, pp. 1758-1767, Apr. 7, 2009.

[10] M. P. Nash, A. Mourad, R. H. Clayton, P. M. Sutton, C. P. Bradley, M. Hayward, D. J. Paterson, and P. Taggart, "Evidence for multiple mechanisms in human ventricular fibrillation," Circulation, vol. 114, pp. 536-542, Aug. 8, 2006.

[11] Y. Chen, M. P. Nash, X. B. Ning, Y. L. Wang, D. J. Paterson, and J. Wang, "Multifractal ECG mapping of ventricular epicardium during regional ischemia in the pig," IEEE Trans. Biomed. Eng., vol. 53, no. 10, pp. 19201925, Oct. 2006.

[12] J. Kneller, R. Q. Zou, E. J. Vigmond, Z. G. Wang, L. J. Leon, and S. Nattel, "Cholinergic atrial fibrillation in a computer model of a two-dimensional sheet of canine atrial cells with realistic ionic properties," Circulation Res., vol. 90, pp. E73-E87, May 17, 2002.

[13] S. Nattel, B. Burstein, and D. Dobrev, "Atrial remodeling and atrial fibrillation mechanisms and implications," Circulation Arrhythmia Electrophysiol., vol. 1, pp. 62-73, Apr. 2008.

[14] K. Shivkumar, N. Lellouche, E. Buch, A. Celigoj, C. Siegerman, D. Cesario, C. De Diego, A. Mahajan, N. G. Boyle, I. Wiener, and A. Garfinkel, "Functional characterization of atrial electrograms in sinus rhythm delineates sites of parasympathetic innervation in patients with paroxysmal atrial fibrillation," J. Amer. Coll. Cardiol., vol. 50, pp. 1324-1331, Oct. 2, 2007.

[15] M. G. Rosenblum, A. S. Pikovsky, and J. Kurths, "From phase to lag synchronization in coupled chaotic oscillators," Phys. Rev. Lett., vol. 78, pp. 4193-4196, Jun. 2, 1997.

[16] Z. Chen, K. Hu, H. E. Stanley, V. Novak, and P. C. Ivanov, "Crosscorrelation of instantaneous phase increments in pressure-flow fluctuations: Applications to cerebral autoregulation," Phys. Rev. E, vol. 73, art. no. 031915, Mar. 2006.

[17] P. Sanders, M. K. Stiles, and G. D. Young, "Virtual anatomy for atrial fibrillation ablation," J. Cardiovasc. Electrophysiol., vol. 17, pp. 349351, Apr. 2006.

[18] D. M. Wei, W. J. Lu, C. W. Yang, Z. X. Fang, X. P. Liu, and X. Zhu, "Implementation of a novel interpolating method to epicardial potential mapping for atrial fibrillation study," Comput. Biol. Med., vol. 40, pp. 456-463, Apr. 2010.
[19] M. Mansour, R. Mandapati, O. Berenfeld, J. Chen, F. H. Samie, and J. Jalife, "Left-to-right gradient of atrial frequencies during acute atrial fibrillation in the isolated sheep heart," Circulation, vol. 103, pp. 26312636, May 29, 2001.

[20] S. Verheule, E. Wilson, S. Banthia, R. H. Everett, S. Shanbhag, H. J. Sih, and J. Olgin, "Direction-dependent conduction abnormalities in a canine model of atrial fibrillation due to chronic atrial dilatation," Amer. J. Physiol.-Heart Circulatory Physiol., vol. 287, pp. H634-H644, Aug. 2004.

[21] M. Costa, A. L. Goldberger, and C.-K. Peng, "Multiscale entropy analysis of biological signals," Phys. Rev. E, vol. 71, art. no. 021906, Feb. 2005.

[22] Y. H. Chen, L. Liang, Q. Pan, Y. Liu, H. Z. Chen, J. Li, R. Brugada, P. Brugada, K. Hong, G. J. Perez, C. M. Zhao, J. Qi, Y. Y. Zhang, L. Y. Peng, and L. Li, "High sensitivity of the sheep pulmonary vein antrum to acetylcholine stimulation," J. Appl. Physiol., vol. 105, pp. 293-298, Jul. 2008.

[23] B. Blasius and L. Stone, "Chaos and phase synchronization in ecological systems," Int. J. Bifurcation Chaos, vol. 10, pp. 2361-2380, Oct. 2000.

[24] E. Keogh, K. Chakrabarti, M. Pazzani, and S. Mehrotra, "Dimensionality reduction for fast similarity search in large time series databases," Knowl. Inf. Syst., vol. 3, pp. 263-286, 2001.

[25] A. G. Kleber and Y. Rudy, "Basic mechanisms of cardiac impulse propagation and associated arrhythmias," Physiol. Rev., vol. 84, pp. 431-488, 2004.

[26] C. E. Shannon, "A mathematical theory of communication," Bell Syst. Tech. J., vol. 27, pp. 623-656, 1948.

[27] D. Dobrev, A. Friedrich, N. Voigt, N. Jost, E. Wettwer, T. Christ, M. Knaut, and $\mathrm{U}$. Ravens, "The $\mathrm{G}$ protein-gated potassium current $\mathrm{I}(\mathrm{K}, \mathrm{ACh})$ is constitutively active in patients with chronic atrial fibrillation," Circulation, vol. 112, pp. 3697-3706, Dec. 13, 2005.

[28] L. Liu and S. Nattel, "Differing sympathetic and vagal effects on atrial fibrillation in dogs: Role of refractoriness heterogeneity," Amer. J. Physiol., vol. 273, pp. H805-H816, Aug. 1997.

[29] J. H. Burn, E. M. V. Williams, and J. M. Walker, "The effects of acetylcholine in heart-lung preparation including the production of auricular fibrillation," J. Physiol., vol. 128, pp. 277-293, 1955.

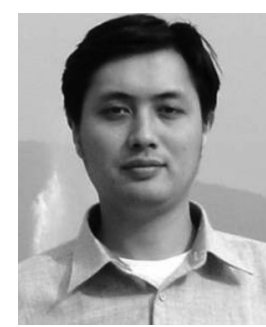

Ying Chen was born in Nanjing, China, in 1976. He received the B.S. degree in electronics and information systems and the Ph.D. degree in acoustics from Nanjing University, Nanjing, in 1994 and 2003, respectively.

He is currently in the Department of Electronic Science and Engineering, Nanjing University. His current research focuses on biomedical signal processing, and nonlinear time series analysis of electrocardiograms with regard to atrial fibrillation and ischemia.

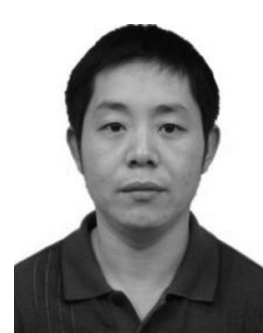

Zhong Wu was born in Xingtai, China, in 1971. He received the M.D. degree in cardiothoracic surgery from Sichuan University, Sichuan, China, in 2002.

$\mathrm{He}$ is currently a Deputy Chief Surgeon in the Department of Thoracic and Cardiovascular Surgery, Nanjing Drum Tower Hospital, The Affiliated Hospital of Nanjing University Medical School, Nanjing, China. His clinical research involves surgical treatment of valve disease, coronary heart disease, congenital heart disease, etc. His research mainly focuses on surgical treatment of atrial fibrillation and 


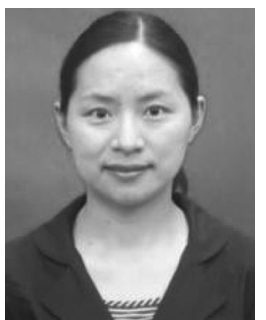

Cuiwei Yang was born in Yueyang, China, in 1969. She received the B.Sc. degree in biomedical engineering from Zhejiang University, Zhejiang, China, in 1992, the M.Sc. degree from the Huazhong University of Science and Technology, Hubei, China, in 1995, and the Ph.D. degree in biomedical engineering from Fudan University, Shanghai, China, in 2008.

She is currently an Associate Professor at Fudan University. Her research interests include epicardial mapping and cardiac electrophysiology.

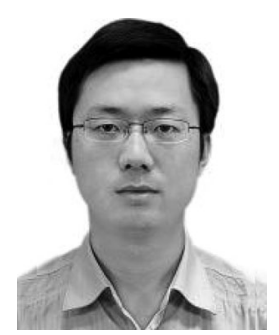

Jun Shao was born in Nantong, China, in 1979. He received the M.D. degree in surgery from Nanjing University, Nanjing, China, in 2008.

$\mathrm{He}$ is currently an Attending Physician in the Department of Thoracic and Cardiovascular Surgery, Nanjing Drum Tower Hospital, The Affiliated Hospital of Nanjing University Medical School, Nanjing. His clinical research involves surgical treatment of valve disease, coronary heart disease, congenital heart disease, etc. His research mainly focuses on dissection of aorta, atrial fibrillation, and their mech-

anisms.

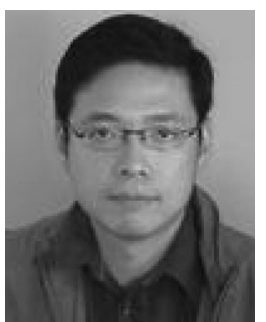

Kelvin Kian Loong Wong was born in Singapore in 1976. He received the B.Eng. degree from Nanyang Technological University, Singapore, the MApplIT degree from the University of Sydney, Sydney, N.S.W., Australia, and the Ph.D. degree from the University of Adelaide, Adelaide, S.A., Australia.

$\mathrm{He}$ is currently a Research Fellow of biomedical engineering at RMIT University, Melbourne, Vic., Australia, and is involved in the development of new medical image processing techniques and medical image velocimetry systems for the study of cardiovascular flow. His research interests include biofluid mechanics and medical image processing.

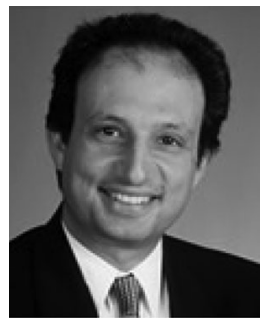

Derek Abbott (F'05) was born in London, U.K., on May 3, 1960. He received the B.Sc. degree (Hons.) in physics from the Loughborough University of Technology, Loughborough, U.K., in 1982, and the Ph.D. degree (with commendation) in electrical and electronic engineering from the University of Adelaide, Adelaide, S.A., Australia, in 1995, under the supervision of K. Eshraghian and B. R. Davis.

He has led a number of research programs in the imaging arena, ranging from the optical to infrared to millimeter wave to T-ray (terahertz gap) regimes. From 1978 to 1986 , he was at the GEC Hirst Research Centre, London, where he was involved in research on the area of visible and infrared image sensors. His expertise spans very large scale integration design, optoelectronics, device physics, and noise, working with nMOS, CMOS, silicon on sapphire, chargecoupled device, GaAs, and vacuum microelectronic technologies. On migration to Australia, he joined Austek Microsystems, Technology Park, S.A., in 1986. Since 1987, he has been with the University of Adelaide, Adelaide, S.A., where he is currently a Full Professor in the School of Electrical and Electronic Engineering and formerly the Director of the Centre for Biomedical Engineering. He has appeared on national and international television and radio and has also received scientific reportage in New Scientist, The Sciences, Scientific American, Nature, The New York Times, and Sciences et Avenir. He holds more than 300 publications/patents and has been an Invited Speaker in more than 80 institutions, including Princeton, NJ; MIT, MA; Santa Fe Institute, NM; Los Alamos National Laboratories, NM; Cambridge, U.K.; and EPFL, Lausanne, Switzerland. He is the Co-Editor of Quantum Aspects of Life (London, U.K.: Imperial College Press) and Stochastic Resonance (Cambridge, U.K.: Cambridge Univ. Press).

Dr. Abbott is a Fellow of the Institute of Physics (IOP), with honorary life membership. He won the General Electric Company Bursary in 1977, the Stephen Cole the Elder Prize in 1998, the E. R. H. Tiekink Memorial Award in 2002, The International Society for Optical Engineers Scholarship Award for Optical Engineering and Science in 2003, the South Australian Tall Poppy Award for Science in 2004, and the Premier's SA Great Award in Science and Technology for outstanding contributions to South Australia in 2004. He has served as an Editor and/or Guest Editor for a number of journals including the IEEE JOURNAL OF SOLID-STATE CIRCUITS, Chaos (AIP), Smart Structures and Materials (IOP), Journal of Optics B (IOP), Microelectronics Journal (Elsevier), Fluctuation Noise Letters (World Scientific), and is currently on the Editorial Board of the PROCEEDINGS OF THE IEEE. He has served on a number of IEEE technical program committees, including the IEEE Asia Pacific Conference on Circuits and Systems and the IEEE Gallium Arsenide Integrated Circuit Symposium. 